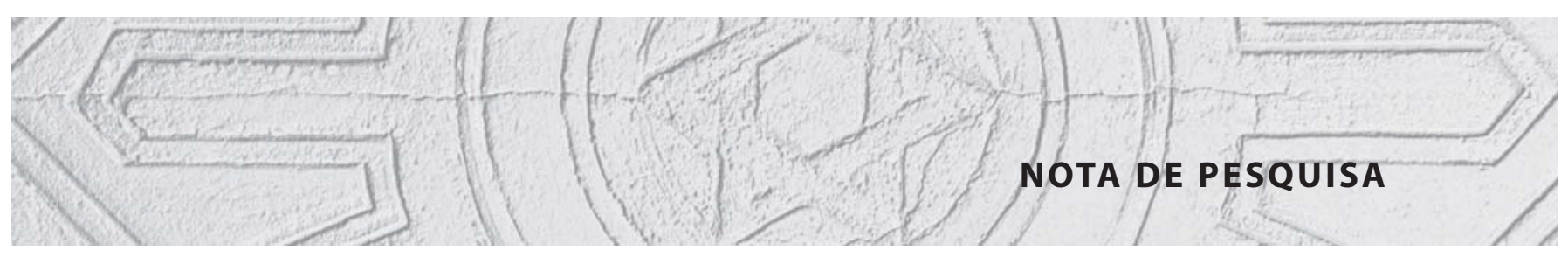

\title{
Sciences et État: note critique sur quelques propositions théoriques
}

\author{
Sciences and the State: a critical note on \\ some theoretical propositions
}

\section{Jérôme Lamy}

Postdoctorant Student, Laboratoire Interdisciplinaire Solidarités,

Sociétés,

Territoires (UMR CNRS 5193)

Centre d'Anthropologie Sociale/ Université Toulouse II.

Maison de la Recherche - 5 Allées

A. Machado

31058 - Toulouse Cedex 9

jerome.lamy@laposte.net

\section{Arnaud Saint-Martin}

Research Fellow, CNRS, Laboratoire Printemps (UMR CNRS 8085)

Université de Versailles Saint-

Quentin-en-Yvelines.

47, Boulevard Vauban

78047 - Guyancourt Cedex

arnaud.saint-martin@uvsq.fr
LAMY, Jérôme; SAINT-MARTIN, Arnaud. Sciences et État: note critique sur quelques propositions théoriques. História, Ciências, Saúde Manguinhos, v.18, n.4, out.-dez. 2011, p.1159-1168.

\section{Résumé}

L'effusion théorique au sein des science and technological studies rend parfois difficile la saisie de lignes directrices ou de points d'appui stables. La question des rapports entre pratiques scientifiques et État offre l'occasion de mesurer les écarts entre différents corpus théoriques. Dans cet article, nous avons considéré trois propositions théoriques récentes. La new production of knowledge écarte, au nom d'un soubassement idéologique néolibéral, la notion d'État de son horizon conceptuel. La théorie de l'acteur-réseau, dissolvant les structures instituées dans l'enchaînement des acteurs, ignore la forme-État. Enfin, les régimes de savoirs accordent une place importante à l'État en tant que ressource pour l'agencement de la production savante.

Mots clés: sciences; État; libéralisme; acteur-réseau; régimes.

\section{Abstract}

The profusion of theories within science and technological studies sometimes makes it hard to find lines of direction or stable points of support. The question of the relations between scientific practices and the state affords an opportunity to appraise the differences between theoretical corpi. The present article explores three recent theoretical propositions. In the name of a neoliberal ideological foundation, the new production of knowledge has eliminated the notion of State from its conceptual horizon. Actor-network theory, suspending the structures instituted within the chain of actors, ignores the state-form. Lastly, regime of knowledge theory accords the state a special role as a source in managing the production of knowledge.

Keywords: sciences; state; liberalism; actor-network; regimes. 
$\mathrm{L}$ es science and technological studies ont toujours tendu à miser nolens volens sur le renouvellement permanent de leur dispositif théorico-conceptuel. Ce bouillonnement ininterrompu opère comme une dynamique de cet ensemble de recherches sur les sciences, souvent très éclatées dans ses intentions comme dans ses méthodes. Comment repérer des lignes directrices, des points d'appuit relativement stables dans cette effusion de concepts, de modèles, de schémas théoriques? La question des rapports entre d'une part les pratiques scientifiques et, de l'autre, l'État et toutes les instances gouvernementales en général constitue pour nous un prisme d'analyse possible. Il donne la possibilité de mesurer avec une certaine acuité les positionnements d'un certain nombre de propositions dans le domaine des science and technological studies. C'est à tout le moins une hypothèse qu'il s'agit de vérifier. Comment? D'abord, en se donnant un corpus de propositions assez explicites pour autoriser ce coup de sonde. Ensuite, en repérant la manière dont les modèles proposés intègrent (ou non) la dialectique sciences/État, on peut se donner les moyens de construire un cadre d'intelligibilité sur au moins deux fronts: premièrement, il s'agit de comprendre les présupposés politiques des science and technological studies quant à la place de l'État dans l'ordre des pratiques scientifiques et, deuxièmement, d'envisager les outils théoriques les plus 'performants' pour saisir cette même articulation. Nous montrerons notamment comment la critique du déploiement néolibéral depuis les années 1970 ainsi que, plus généralement, la remise en question des pratiques intégrant les notions, certes polysémiques de service public ou d'intérêt général, impose la notion d'État, tout aussi flottante dans ses définitions contradictoires, comme opérateur d'analyse particulièrement efficace.

Nous nous attacherons, dans cette courte note, à retracer les relations entre sciences et État telles que les envisagent trois propositions théoriques: la "nouvelle production des savoirs", proposée par Michael Gibbons, Camille Limoges, Helga Nowotny, Simon Schwartzman, Peter Scott, Martin Trow (Gibbons et al., 1994), la théorie de l'acteur-réseau, portée par Bruno Latour et enfin les "régimes de savoirs" proposés par Dominique Pestre (2003). Pour chacun de ces ensembles conceptuels plus ou moins vertébrés, nous envisagerons son positionnement (indissociablement) épistémique et politique quant au rôle de l'État dans la production des savoirs, ses relations avec les propositions politiques dominantes et les points aveugles que celles-ci ne manquent pas de véhiculer.

\section{Une"nouvelle production des savoirs"}

Dans le si commenté The new production of knowledge, Gibbons et al. (1994) conçoivent les rapports entre la science et la société dans les sociétés contemporaines. Cette approche est approfondie dans l'ouvrage plus récent, Repenser la science (Nowotny, Gibbons, Scott, 2003). Le groupe de chercheurs à l'origine du paradigme de la new production of knowledge introduit une distinction entre les 'modes' 1 et 2 de production des connaissances. Le schème est désormais bien connu. Dans le glossaire ayant valeur de toolkit, on apprend que le mode 1 est un "complexe d'idées, de méthodes, de valeurs et de normes" dont le développement résulte de la diffusion d'un "modèle newtonien de la science" (Gibbons et al., 1994, p.166). La science est un mets rare échangé sur un marché fermé et protégé par les remparts du champ universitaire. Le savoir est produit et consommé par et pour les spécialistes 
des disciplines aux frontières nettement tracées. Le mode 2 émerge par contraste avec le mode 1 après la Seconde Guerre mondiale. La rupture n'est pas franche, puisque les deux modes cohabitent. Néanmoins, le nouveau régime tend à supplanter le premier, en raison notamment de son efficacité économique. La production des connaissances atteint vite un niveau élevé de complexité. Les producteurs se soucient des 'applications'. L'interaction entre les industries, le système universitaire et l'État est de plus en plus étroite. Les sites de production sont 'multifonctionnels' et ne se cantonnent plus au seul périmètre de l'université. La recherche accomplie dans les laboratoires de recherche et développement exemplifie un modèle marchand de développement de la science. Dans ce contexte de brouillage des anciens repères, les valeurs de transdisciplinarité, de fluidité, de flexibilité priment sur les critères 'disciplinaires' de l'évaluation par les pairs; la validité d'une innovation se mesure à l'aune de son utilité sociale et de standards de qualité contrôlée. L'appel au mode 1 (de certains scientifiques) relève alors d'une forme de nostalgie et/ou de résistance au changement. En somme, elle est en phase avec l'émergence des sociétés du 'savoir socialement distribué', dans lesquelles les savoirs sont impurs et hybrides per se.

Nous ne reviendrons pas sur le caractère simpliste de certaines affirmations, qu'infirment nombre d'études d'histoire des sciences (Godin, Gingras, 1998; Pestre, 2003; Shinn, 1999; Weingart, 1997). Les points suivants nous paraissent importants. Sur le plan théorique, l'objectif affiché de la new production of knowledge est de dégager les propriétés d'une forme générale de production des savoirs. L'approche souligne la variété des configurations socioinstitutionnelles de la science contemporaine. Par exemple, le 'champ vaste d'interconnexions' entre les différents sites de production est le signe d'une extraordinaire complexité de la recherche contemporaine (Gibbons et al., 1994, p.22). Concrètement, le modèle entend démontrer la coalescence d'éléments hétérogènes dans un mode idéal et syncrétique. Pourtant, l'emphase sur l'hétérogénéité est paradoxalement neutralisée par une description moniste du système de la science contemporaine. La new production of knowledge procède par accumulation d'éléments très généraux en vue de soutenir une thèse classique (très mode 1, pourrait-on dire) sur l'unicité organique des institutions de la science. Que les interactions entre la science, l'État et l'industrie soient 'plus' étroites n'est finalement pas un facteur crucial. Cette assertion revient à dire que, malgré tout, il y avait bien interaction entre les sphères etatique, universitaire et industrielle avant l'éclosion providentielle du mode 2. L'autonomie postulée du mode 1 suppose en effet qu'il y ait un type de connexion État/université qui garantisse celle-ci.

La new production of knowledge ne fait pas mystère de son adhésion à un programme libéral de management des innovations. Le dernier chapitre de l'ouvrage est clair de ce point de vue. Les évolutions de l'économie globale ont des effets directs sur la production du savoir dans le mode 2. La commercialisation des applications s'inscrit dans une économie des biens scientifiques fondée sur la compétition et en nouvelles formes de collaboration. Les scientifiques sont des entrepreneurs engagés dans une 'course' au profit (Gibbons et al., 1994, p.56). Pour les entreprises innovantes, la connaissance est une 'ressource économique'. L'ambition des auteurs de la new production of knowledge est de penser les conditions d'une croissance de ces secteurs de la recherche privée. Par voie de conséquence, cette perspective normative assigne un certain rôle à l'État. L'immixtion des gouvernements 
dans ces activités peut contrevenir à la bonne marche des activités des nouveaux producteurs de la connaissance. Les performances des politiques gouvernementales d'aide au développement industriel ou aux transports, par exemple, sont considérées comme ambiguës (p.136). Il est important que les représentants des gouvernements se mettent en retrait dans les processus décisionnaires. Dans cette économie, ils doivent être des 'intermédiaires honnêtes' (honest brokers) entre les innovateurs, les régulateurs, les traders, etc. Dès lors, il ne fait aucun doute que la République de la science (mode 1) s'essouffle devant la montée des forums hybrides et des arènes politiques transcommunautaires (mode 2), dans lesquels le savoir est l'objet d'appropriations multiples (p.162). Cette focalisation sur la libéralisation des échanges est stratégique. L'État est impensé pour de bonnes raisons. Dans Repenser la science (Nowotny, Gibbons, Scott, 2003), il est affirmé que l’État-nation est une institution de mode 1. En d'autres termes, il est une survivance appelée à disparaitre dans la société contemporaine. Ce constat apparaît fondé empiriquement. L'application de critères de 'rentabilité' dans l'évaluation des hôpitaux et des universités serait le symptôme d'un effacement des services publics de l'État-nation; le discours marchand affaiblit l'“éthique du service public" (p.45). De même, les établissements de recherche gouvernementaux institutionnalisés dans le but d'accomplir des recherches d'intérêt national sont refondus suite à des privatisations en chaîne. Les services proposés s'adaptent aux exigences de nouvelles 'clientèles'.

Finalement, tout ce qui de près ou de loin se rattache à l'État est donc écarté au nom d'un nouveau style de management et d'une évolution inexorable de l'économie mondialisée. Chemin faisant, la new production of knowledge s'interdit l'analyse de sciences contemporaines qui ne relèvent ni du mode $1 \mathrm{ni}$ du mode 2 . Des sciences qui doivent leur existence (sous la forme d'organisations) et leur champ d'intervention à l'État.

\section{La traduction de l'État dans les réseaux sociotechniques}

La théorie de l'acteur-réseau est une approche possible du régime de la production scientifique. La parenté avec la new production of knowledge est étroite si l'on considère la question de l'État. Dans les deux cas, il est en effet question d'une 'co-évolution' de la science et de la société à laquelle l'État ne contribue que dans une certaine mesure.

L'étude que Latour $(1984,1994)$ consacre au pasteurisme est une bonne entrée en matière. L'auteur prétend resituer Pasteur 'dans son époque'. Le savant fait œuvre de démiurge. À lui seul, il parvient en effet à enrégimenter les "branches industrielles, l'élevage, l'hygiène, l'Académie des sciences, ses collègues, les microbes et les épidémies" (Latour, 1994, p.66). Le réseau est un homme pensant, nous dit Latour. Le laboratoire 'soulève' un monde nouveau et par l'effet d'un mécanisme trouble de traduction, change la société française de fond en comble. La France n'est plus la même sous l'administration de la science pasteurienne. Cette reconstruction est saisissante. Néanmoins, sans même se prononcer quant à la validité de ce scénario historique, celui-ci illustre une certaine conception du 'non rapport' de la science à l'État. La III République est ingrate à l'endroit du savant, à qui les crédits manquent pour l'établissement de son laboratoire. Si donc Pasteur associe son entreprise aux préoccupations des hommes politiques, c'est simplement en vue de maximiser ses chances 
d'obtenir des fonds. L'État n'est qu'un bailleur de fonds. Il ne se manifeste que sous la forme d'un ectoplasme imperceptible. ${ }^{1}$ Pourtant, précise Latour, c'est toute la société qui est transfigurée. Quid du régime politique qui met à l'épreuve le pastorisme?

L'approche en termes de traduction valorise donc une forme de coproduction de la réalité. Aux rapports dyadiques simplistes du type "la Science est influencée par la politique d'un gouvernement" (et réciproquement), la théorie de l'acteur-réseau substitue une technique de description des processus de traduction des éléments hétérogènes qui composent les réseaux socio-techniques (i.e. le seul véritable 'objet' qu'il faille retenir selon la théorie de l'acteur-réseau). Dans cette continuité, la critique antidifférenciationniste de la notion de société va de pair avec une déconstruction des catégories courantes utilisées pour penser le politique. Elle est au fondement du projet d'une 'épistémologie politique' porté par la théorie de l'acteur-réseau. Dans son essai Nous n'avons jamais été modernes, Latour (1991) dévoile les fondements de la 'constitution moderne' de l'Occident. L'auteur veut faire tomber les masques sans pour autant céder aux lubies d'une pensée critique dénonciatrice. D'après lui, les concepts de représentation politique et de représentation scientifique sont des illusions bien fondées formant les "deux branches du même gouvernement" moderne (p.48). Le Léviathan hobbesien est une "créature artificielle" qui prend son sens dans un "nouveau régime où la connaissance égale le pouvoir" (p.32). Le commerce des individus repose ainsi sur la délégation du collectif à une représentation transcendante et moniste. Or, selon Latour, tout cela n'est qu'artifice. La société est l'assemblage des hommes par les hommes, pour les hommes. Ne comptent que les connexions entre les actants, humains et non humains. La théorie de l'acteur-réseau refuse le partage entre ce qui relève de la politique d'un côté, de la science de l'autre. Dans son étude du programme de recherche lancé par Frédéric Joliot au Collège de France, Latour (2001) considère qu'il est essentiel de tenir en son entier le travail de 'traduction' auquel se livre le physicien. Tous les actants sont pertinents, de la matière première de l'uranium aux ministres du gouvernement. La mobilisation est multifocale. Selon Latour, en effet, "maintenir le ministre et les neutrons engagés dans le même projet, les faire agir, les discipliner, n'étaient pas pour Joliot des tâches vraiment distinctes. Les deux lui étaient nécessaires". (p.93; souligné par l'auteur) Contre les clichés d'une histoire des sciences tour à tour 'internaliste' ou 'externaliste', Latour traduit le politique et le scientifique dans un imbroglio socio-technique. L'État est un simple réservoir d'alliés que Joliot parvient à convaincre en touchant la corde sensible de l'intérêt de la nation.

La théorie de l'acteur-réseau dissout dans le bain d'acide des réseaux les "objets traditionnels de la théorie sociale". L'entité État en est une des composantes (Latour, 1991, p.164). L'anthropologie 'symétrique' propose des descriptions 'réalistes' de ses objets. C'est une simple question d'échelle et d'attention au détail pratique. Pour rendre compte de la constitution d'un État, par exemple, plutôt que de partir de l'intuition d'une entité surplombante et contraignante, il convient de tirer les fils mêlés du "réseau de statistiques et de calculs, de bureaux et d'enquêtes" (p.166). Il en ira ainsi si l'on choisit d'examiner les relations qu'entretiennent les deux grands fétiches de la Modernité, à savoir la science et l'État. L'approche est en quelque sorte 'tactile'. L'anthropologue ne veut pas voir, il veut toucher. On suit la réalité des groupes à travers la matérialité des modes de regroupement 
continuel. Il n'est plus d'arrière-monde. Il faut suivre le fil des interconnexions entre les actants à travers les "longues chaînes de médiation" (Latour, 2006, p.125). Pas de bureaucratie abstraite du gouvernement, mais des bureaucrates, des 'paperassiers' qui traduisent en dossiers les multiples composants d'un réseau (Latour, 1989, p.418-423). Parler d'un rapport entre la science et l'État, c'est finalement commettre une pétition de principe, c'est-à-dire jouer avec des mots. L'attention portée sur la circulation et le mouvement dans les processus de traduction conduit immanquablement à une dissolution de l'artifice de l'État.

Penser les rapports entre la science et l'État dans le cadre de la théorie de l'acteur-réseau apparaît comme un non sens. L'État est invisible. Et c'est pour cette raison qu'il faut éviter d'utiliser cette catégorie d'analyse (elle est l'explandum, pas l'explanans). La bonne approche consiste à tracer un acteur-réseau, à produire de la trace à partir des traces tangibles. Cette conception iconoclaste des rapports entre la science et le politique repousse les cadres d'analyse qui empruntent par exemple à la sociologie des organisations.

\section{Une histoire sociale et culturelle des régimes successifs de savoirs}

Dominique Pestre a proposé une manière d'envisager l'évolution globale de la science qui a pour point de départ une critique argumentée de la new production of knowledge. Il conteste la pertinence historique des modes 1 et 2 . Le mode 1 n'a, selon Pestre (2003, p.18), jamais existé en tant que tel (c'est un type idéal), puisque les pratiques scientifiques ont toujours été associées aux décisions politiques, aux intérêts économiques, et aux recherches militaires. Il n'existe pas de coupure nette entre une conception de la science 'pure', ancienne, totalement dégagée du jeu social et une forme hybridée et réticulaire de la science, plus récente. En somme, pour Pestre, une lecture correcte des grandes transformations de la science à la lumière de la new production of knowledge devrait permettre de conclure à la permanence du mode 2. Toutefois, l'historien propose un autre schéma conceptuel pour penser l'évolution générale des pratiques savantes et de leur place dans la société. Il fonde la notion de régime de production, de régulation et d'appropriation des savoirs (Pestre, 2003, 2006), qui correspond à une conception plurielle de l'activité scientifique impliquant des productions écrites et techniques, des pratiques instrumentales ou calculatoires, mais aussi des valeurs, des normes, des règlements, des formes d'organisation institutionnelles, des interactions multiples avec les sphères politiques, économiques et juridiques. (Pestre, 2003, p.34). Les régimes de savoirs fournissent une grille d'analyse séquentialiste des évolutions de la science. Un régime de savoirs correspond, à un moment donné, à un équilibre précis entre les divers éléments qui le composent. Il désigne une articulation particulière, un agencement spécifique de l'ensemble de ses dimensions constitutives. Les régimes de savoirs permettent de dégager des périodes successives pour lesquelles les modes d'organisation, de production et de régulation des pratiques scientifiques sont cohérentes et correspondent à un compromis social stabilisé. Ce sont donc des séquences historiques qui se succèdent et reposent sur une analyse contextualisée de la place des savoirs dans le jeu social. C'est à l'intérieur d'un régime englobant que la hiérarchie entre les savoirs (la hiérarchie disciplinaire, par exemple) est instituée (Pestre, 2006, p.105). 
Même s'il s'intéresse plus particulièrement au régime de savoirs apparu depuis les années 1970, Pestre fournit des repères sur les régimes précédents et leurs caractéristiques. Les XVI éme et XVII éme siècles sont marqués par des changements sociaux, économiques, politiques et militaires majeurs: la navigation, la découverte de nouveaux territoires, la multiplication des conflits guerriers ont permis de faire émerger des pratiques savantes centrées à la fois sur les formes appliquées (comme la construction d'instruments) et sur le service des Etats dans la perspective d'une domination géopolitique et d'un meilleur contrôle des espaces (Pestre, 2003, p.24-25). Le siècle des Lumières poursuit en quelque sorte cette double inscription des savants dans l'ordre économique marchand et dans les actions politiques liées aux intérêts des souverains. En France, les savoirs académiques et les savoirs pratiques ne sont pas disjoints, et surtout ils visent à repenser l'organisation de la société en s'appuyant sur les principes des Lumières (p.27). Le XIX éme siècle est quant à lui marqué par une circulation intense des acteurs de la science entre monde académique et monde industriel (p.29). C'est également à cette époque que se constitue, dans le monde académique, la prétention à établir une 'science pure' dégagée des contraintes socio-économiques. Pour Pestre, si cette ambition structure bien l'argumentaire des savants en quête d'autonomie, elle n'en est pas moins frappée d'irréalité. En effet, la science n'est pas pure, elle s'insère toujours dans des situations sociales et politiques. Les discours sur la science pure sont des "constructions sociales à finalité politique" (Pestre, 2006, p.97).

Pestre poursuit cette description des régimes de savoirs successifs en se focalisant principalement sur la période qui court de la III République jusqu'au crépuscule des Trente Glorieuses. Il consacre une grande partie de son analyse à détailler la nouvelle place de l'État dans l'ordre social (Pestre, 2003, p.40-50). En Occident, à la fin du XIX éme siècle, la puissance publique est d'abord perçue comme un ciment de l'identité nationale. L'État prend désormais à son compte la question du bien public et collectif. Il déploie donc des modes de régulation et des formes d'intervention qui renforcent sa présence sur les différentes scènes sociales.

Pestre souligne que les formes d'auto-organisation, même si elles se marginalisent quelque peu, ne disparaissent pas totalement. Les sociétés occidentales connaissent également, de 1870 à 1970, des formes originales de production matérielle. La massification des échanges et la standardisation des procédés de fabrication sont sous-tendues par une exigence de rationalité érigée en principe d'organisation et de production. La science participe activement à la rationalisation des sociétés. Elle intervient directement dans la gestion industrielle et la recherche militaire dans le champ d'action des Etats-nations (Pestre, 2006, p.107). Caractérisée par le réductionnisme, le pragmatisme, la formalisation, et la plus forte présence des savoirs appliqués dans les cénacles académiques, la science, de 1870 à 1970, est très fortement intégrée dans l'organisation et la gestion des nations. Elle s'inscrit dans les nouvelles formes de relations qui se construisent entre État et industrie. Avec l'industrie, l'État 'nationalise' la science (e.g. le Centre National de la Recherche Scientifique, le Commissariat à l'Energie Atomique). Le XX éme siècle voit la création d'institutions technoscientifiques d'État. La science constitue un moyen d'action (Pestre, 2004).

Pestre (2003, p.77-118) repère un dernier régime de savoirs, apparu dans les années 1970. Il souligne que les formes d'organisation et de régulation sociales évoluent avec la plus 
grande financiarisation de l'économie, la condensation rapide des théories néo-libérales dans la conduite politique des nations et le recul des formes de solidarité étatisées. La rupture avec le précédent équilibre social entraîne l'avènement d'un nouveau régime de savoirs dans lequel le marché occupe une place prépondérante parallèlement à la double privatisation des sciences et de l'État.

Les régimes de savoirs fournissent donc une grille d'analyse globale des évolutions de la science, en caractérisant des successions d'équilibres et de compromis sociaux. Ils ne visent pas à discriminer les formes de pratiques scientifiques qui peuvent coexister et déployer des modes de production et d'organisation différents. Leur objectif est autre: historiciser les articulations entre les savoirs et la société; dépasser les simplifications à visées normatives de la new production of knowledge. A la différence de Latour à qui il emprunte nombre d'analyses, Pestre (Pestre, 2006) considère systématiquement l'État comme un acteur majeur dans les redéfinitions des diverses "économies de la connaissance" (p.103) qui se succèdent dans l'histoire. Il envisage ses mutations et les conséquences pour la structuration des pratiques scientifiques. Néanmoins, l'appropriation des savoirs est considérée sous l'angle de l'instrumentalisation. L'État et l'industrie s'approprient certains savoirs scientifiques en vue d'élargir leur champ d'action à dessein. Si bien que la science est seulement au service de l'État techno-scientifique. ${ }^{2}$

Pestre a intégré, sous forme d'une critique latente, l'érosion néolibérale des cadres étatiques. Il envisage l'État moins comme un opérateur scientifique que comme un réservoir de potentialités pour d'autres acteurs.

\section{Pour conclure provisoirement}

Les trois modèles théoriques envisagent avec des intensités très variables l'articulation sciences/État. La new production of knowledge marquée par l'éviction de la chose commune du projet néolibérale, écarte nettement l'État de son horizon conceptuel. Dans ce régime moderne du capitalisme managérial, la science apparait comme une activité fluide, dégagée des contraintes structurelles d'un étatisme considéré comme les vestiges d'un autre âge. En portant cette prophétie autoréalisatrice de pratiques scientifiques se déployant dans des espaces sociaux vides de formes communes, la new production of knowledge s'impose clairement en projet politique néolibéral. La théorie de l'acteur-réseau procède différemment, en ce sens qu'elle ne repose pas (explicitement à tout le moins) sur une intention politique claire: son horizon d'attente épistémique procède surtout du coup d'éclat permanent. La constitution d'un réseau d'acteurs, enchaînés les uns aux autres dans un monde social résolument plat ne permet pas d'intégrer la question de l'État. Cette notion paraît même incongrue dans le projet latourien, puisqu'elle renvoie à des formes d'organisation fatalement réifiées par leur emploi générique. Il ne peut être question d'un État sans acteurs précis et sans articulations: la forme-État est définitivement invisible au prisme analytique de la théorie de l'acteur-réseau. Les régimes de savoirs mise en œuvre par Dominique Pestre intègrent l'État dans leur cadre d'intelligibilité; il est convoqué comme un pur instrument inerte par lui-même. L'inflexion néolibérale est attentivement étudiée, dans une perspective 
critique, et elle permet à Pestre de rappeler la place de prééminence de l'État dans l'agencement de la production savante.

Globalement l'État reste, dans trois grandes propositions théoriques en science and technological studies que nous avons étudiées, un impensé majeur. Evacué par l'impatience néolibérale de la new production of knowledge, invisible dans le réseau des acteurs latouriens et simple instrument dans les régimes de savoirs, l'État n'a pas encore fait l'objet d'un projet conceptuel conséquent pour saisir ses articulations avec les pratiques scientifiques. Dans un prochain article, nous formulerons quelques propositions théoriques visant à systématiser le régime socio-historique travaillant les activités savantes incluses dans le dessein politique du bien commun et du service public. En inscrivant notre démarche dans le cadre de l'approche transversaliste développée par Terry Shinn (Shinn, Ragouet, 2005), nous détaillerons les critères d'une pratique de la science organisée par et pour l'État, centrée sur l'office et cadrée par une trame juridique précise.

\section{NOTES}

${ }^{1}$ Latour ne s'en tient pas à l'exemple français. De la même façon, les expériences télégraphiques de lord Kelvin contribuent à redessiner les contours de l'Empire britannique. Les câbles télégraphiques (i.e. un réseau technique) confèrent à cette entité mystérieuse qu'est l'Empire un début de réalité bien tangible. Ils participent même de sa création (Latour, 2006, p.156).

${ }^{2}$ Cette perspective est répandue. Pour une position relativement similaire, voir Schmandt, Katz, 1986.

\section{REFERENCES}

GIBBONS, Michael et al.

The new production of knowledge: the dynamics of science and research in contemporary societies. Lonon: Sage. 1994.

GODIN, Benoît; GINGRAS, Yves. The place of universities in the system of knowledge production, Research Policy, Amsterdam, v.29, n.2, p.273-278. 1998.

LATOUR, Bruno.

Changer de société, refaire de la sociologie. Paris: Gallimard. 2006.

LATOUR, Bruno.

L'espoir de Pandore. Paris : La Découverte. 2001.

LATOUR, Bruno.

Pasteur, une science, un style, un siècle. Paris: Perrin. 1994.

LATOUR, Bruno.

Nous n'avons jamais été modernes. Paris: La

Découverte. 1991.

LATOUR, Bruno.

La science en action. Paris: Gallimard. 1989.

LATOUR, Bruno.

Les microbes: guerre et paix. Paris: Métailié. 1984.
NOWOTNY, Helga; GIBBONS, Michael;

SCOTT, Peter.

Repenser la science: savoirs et société à l'ère de l'incertitude. Paris: Belin. 2003.

PESTRE, Dominique.

Introduction aux science studies. Paris: La Découverte. 2006.

PESTRE, Dominique (Ed.).

Deux siècles d'histoire de l'armement en France: de Gribeauval à la force de frappe. Paris: CNRS Editions. 2004.

PESTRE, Dominique.

Science, argent et politique: un essai d'interprétation. Paris: INRA Editions. 2003.

SCHMANDT, Jurgen; KATZ, James Everett. The scientific state: a theory with hypothezise. Science, Technology \& Human Values, Cambridge, v.11, n.1, p.41-52. 1986.

SHINN, Terry.

Change or mutation?: reflections on the foundations of contemporary science. Social Science Information, London, v.1, n.38, p.149176. 1999 . 
Jérôme Lamy, Arnaud Saint-Martin

SHINN, Terry; RAGOUET, Pascal.

Controverses sur la science: pour une sociologie transversaliste des activités scientifiques. Paris:

Raison d'Agir. 2005.
WEINGART, Peter.

From 'finalization' to 'mode 2': old wine in new bottles?. Social Science Information, London, v.36, n.4, p.591-613. 1997.

\section{$\rightarrow \rightarrow \rightarrow<<<$}

